\title{
Hydrodynamics of ideal fracton fluids
}

\author{
Kevin T. Grosvenor $\odot,{ }^{1,2}$ Carlos Hoyos $\odot,{ }^{3}$ Francisco Peña-Benitez $\odot,{ }^{1,2, *}$ and Piotr Surówka ${ }^{4,1,2}$ \\ ${ }^{1}$ Max Planck Institute for the Physics of Complex Systems, 01187 Dresden, Germany \\ ${ }^{2}$ Würzburg-Dresden Cluster of Excellence ct.qmat, 01187 Dresden, Germany \\ ${ }^{3}$ Department of Physics and Instituto de Ciencias y Tecnologías Espaciales de Asturias (ICTEA) Universidad de Oviedo, \\ c/ Federico García Lorca 18, ES-33007 Oviedo, Spain \\ ${ }^{4}$ Department of Theoretical Physics, Wroctaw University of Science and Technology, 50-370 Wroctaw, Poland
}

(Received 12 May 2021; revised 12 November 2021; accepted 22 November 2021; published 16 December 2021)

\begin{abstract}
Low-energy dynamics of many-body fracton excitations necessary to describe topological defects should be governed by a novel type of hydrodynamic theory. We use a Poisson bracket approach to systematically derive hydrodynamic equations from conservation laws of scalar theories with fracton excitations. We study three classes of theories. In the first class we introduce a general action for a scalar with a shift symmetry linear in the spatial coordinates, whereas the second one corresponds with a complex scalar, while the third class serves as a toy model for disclinations and dislocations propagating along the Burgers vector. We apply our construction to study hydrodynamic fluctuations around equilibrium states and derive the dispersion relations of hydrodynamic modes.
\end{abstract}

DOI: 10.1103/PhysRevResearch.3.043186

\section{INTRODUCTION}

Recent years have seen a dramatic growth of interest in the study of fracton phases of matter [1,2]. In these phases, the motion of the elementary fractonic excitations is spatially restricted. While earlier spin models with such excitations (e.g., Refs. [3-7]) describe gapped fracton phases, gapless phases have also been proposed in the context of certain spin liquids [8-12], dipole-conserving lattice models [13-17], and quantum elasticity [18-28] (see also Refs. [29-31], where similar structures appear). From an effective field-theory perspective, such gapless phases are of particular interest as they are typically the ones that survive and remain robust at very low energies. The dynamics of such a low-energy regime should be governed by a hydrodynamic theory, which reflects the symmetries and, in consequence, the conservation laws of a fractonic system.

Hydrodynamics offers a phenomenological description of interacting many-body systems. It focuses on a subset of physical quantities that remain conserved at low energies such as the particle number or momentum. Fractonic excitations conserve not only charge but also one or several higher moments of charge. It follows that the low-energy regime is characterized by long thermalization, subdiffusive behavior of systems without momentum conservation, and a different structure of continuity equations. This has already been noticed in models of a scalar field with fracton excitations that

\footnotetext{
*pena@pks.mpg.de
}

Published by the American Physical Society under the terms of the Creative Commons Attribution 4.0 International license. Further distribution of this work must maintain attribution to the author(s) and the published article's title, journal citation, and DOI. Open access publication funded by the Max Planck Society. emerge as a generalization of ordinary superfluids $[32,33]$ or in the dissipative diffusive evolution of charges with restricted mobility [34]. Another class of fractonic fluids arises in the dynamics of topological defects [35].

Topological defects arise in many areas of physics and play an important role in, e.g., superfluids [36], quantum Hall effect [37], liquid crystals [38], and metamaterials [39]. The interpretation of topological defects as fractons is most visible in the language of elastic dualities [40-43] that map elastic displacements to tensor gauge fields and the defects to charges that source the gauge fields. As was first observed in Refs. [10,11], fractonic charges with restricted mobility naturally source tensor gauge fields. Therefore topological defects can be interpreted as fractons. This interpretation allows one to construct low-energy coarse-grained models of defect dynamics based on the underlying symmetries, which are otherwise difficult to obtain and challenging to study from first principles, even numerically. Such an approach was successfully applied to superfluid vortices [44-51].

In this paper, our interest is in the effective field-theory description of fracton fluids. For simplicity, we will only consider nondissipative fluids and leave the study of additional phenomena, such as dissipation, for future work. We follow the approach of Refs. [52-56], starting from a sought-after algebra of symmetries, the corresponding conservation equations, and eventually deriving the hydrodynamic equations of motion for an ideal fluid. We focus on three classes of models: A real scalar theory with a shift symmetry linear in the spatial coordinates that extends previously studied models [34,57,58], a complex scalar theory with a global $U(1)$ and a global vector symmetry previously studied in Refs. [59,60], and a chiral scalar theory that has a Burgers-like vector, constraining the movement of charges. While the nonchiral models are analyzed in general dimensions, the proliferation of the types of defects in spatial dimensions higher than two 
leads us to focus the analysis of the chiral model to two spatial dimensions. The chiral theory can be viewed as a toy model to understand the basic physical properties of manybody disclination dynamics. Given these theories we motivate the corresponding hydrodynamic Poisson structure and the derivative expansion in hydrodynamics. The main outcome of our analysis is that the Poisson structure for fracton systems is the same as in conventional fluids, but the constitutive relations change, thus leading to novel types of hydrodynamic theories. We analyze the corresponding hydrodynamic modes in both theories. Our construction paves the way for a systematic study of transport properties in fracton theories.

\section{HYDRODYNAMIC EQUATIONS FROM POISSON BRACKETS}

Hydrodynamic equations represent the macroscopic conservation laws for quantities preserved during the time evolution of a physical system. Although conceptually simple, the precise form of these equations can be rather challenging to obtain. The difficulty may be associated with the nonlinear terms or the proper inclusion of degrees of freedom in the case of systems with multiple conserved quantities. Therefore, in order to circumvent these difficulties, it is convenient to use the Poisson bracket formulation of hydrodynamics (for a review, see Ref. [55]). This has been successfully applied to various systems and theories, e.g., to superfluids $[52,53]$, liquid crystals [61], and more recently the fractional quantum Hall effect [56]. One starts by postulating the Poisson brackets between conserved densities. Such a postulate can be justified a posteriori by computing corresponding commutators in a microscopic model. As an instructive example, we present a derivation of the conservation laws for a fluid with Galilean symmetries. The relevant degrees of freedom are the number density $\rho(\boldsymbol{x})$, the momentum density $p_{i}(\boldsymbol{x})$, and the entropy density $s(\boldsymbol{x})$. The Poisson brackets for these variables read

$$
\begin{aligned}
\left\{p_{i}(\boldsymbol{x}), \rho(\boldsymbol{y})\right\} & =-\rho(\boldsymbol{x}) \partial_{x^{i}} \delta(\boldsymbol{x}-\boldsymbol{y}), \\
\left\{p_{i}(\boldsymbol{x}), s(\boldsymbol{y})\right\} & =-s(\boldsymbol{x}) \partial_{x^{i}} \delta(\boldsymbol{x}-\boldsymbol{y}), \\
\left\{p_{i}(\boldsymbol{x}), p_{j}(\boldsymbol{y})\right\} & =-\left[p_{j}(\boldsymbol{x}) \partial_{x^{i}}+p_{i}(\boldsymbol{y}) \partial_{x^{j}}\right] \delta(\boldsymbol{x}-\boldsymbol{y}),
\end{aligned}
$$

with all other Poisson brackets vanishing. In the subsequent analysis, we focus on the zero-temperature fluid. The energy density in the fluid is a function of hydrodynamic variables $h=h(\rho, \boldsymbol{p})$. This is a thermodynamic potential whose differential is given by

$$
d h=\mu d \rho+v^{i} d p_{i},
$$

where we have introduced the chemical potential $\mu$ and fluid velocity $\boldsymbol{v}$. Note that we consider a nonstationary fluid configuration. The equations of motion follow from the Poisson brackets of the hydrodynamic variables with the Hamiltonian ${ }^{1}$

$$
\begin{aligned}
\partial_{t} \rho & =\{\rho, H\}=-\partial_{i}\left(\rho v^{i}\right), \\
\partial_{t} p_{i} & =\left\{p_{i}, H\right\}=-\rho \partial_{i} \mu-\partial_{j}\left(v^{j} p_{i}\right)-p_{j} \partial_{i} v^{j},
\end{aligned}
$$

\footnotetext{
${ }^{1}$ Note that many references use different conventions, which results in a time evolution given by $\{H, \cdot\}$.
}

where $H=\int d^{d} x h(\rho, \boldsymbol{p})$. The momentum equation is not yet in the form of a conservation law. However, using the Legendre transform of pressure $d p=\rho d \mu+p_{i} d v^{i}$, we can rewrite it as a momentum flux

$\partial_{t} p_{i}=-\partial_{k}\left(v^{k} p_{i}\right)-\partial_{i} p=-\partial_{k}\left(v^{k} p_{i}+p \delta_{i}^{k}\right) \equiv-\partial_{k} T^{k}{ }_{i}$.

As a result, momentum conservation defines the stress tensor. This is a general form of the hydrodynamic equation in a theory that conserves particle number and momenta. It is valid both for nonrelativistic and relativistic fluids. In order to derive the final form of Euler's equations for a fluid that respects the Galilean symmetry, we need to impose appropriate constraints. At this point, we can directly supplement the Poisson brackets (1) with a specific Hamiltonian or with a generic one whose form is fixed by the appropriate symmetries and thermodynamics. For example, in the Galilean fluid, the momentum density is equal to the mass flux of particles

$$
p_{i}=m \rho v_{i}
$$

where $m$ is the mass of the fluid constituents. Equation (5) can be rewritten as a constraint on the pressure thermodynamic potential

$$
\frac{\partial p}{\partial v_{i}}=m v_{i} \frac{\partial p}{\partial \mu},
$$

with a solution given by $p=p\left(\mu+\frac{1}{2} m v^{2}\right)$. We can interpret this new variable as the chemical potential in the frame moving with the fluid element $\tilde{\mu}=\mu+\frac{1}{2} m v^{2}$. Using this result we can recover the usual form of Euler's equations. The stress tensor now has a manifestly symmetric form

$$
T_{i j}=m \rho v_{i} v_{j}+p \delta_{i j},
$$

and the energy density is equal to

$$
h=\mu \rho+v_{i} p_{i}-p=\frac{1}{2} m \rho v^{2}+\tilde{\mu} \rho-p,
$$

where we can identify the kinetic energy and the internal energy.

As a final step we want to justify our postulate (1) in a microscopic theory. This was first done by Landau in the context of superfluid helium [52]. We start with a Galilean-invariant Lagrangian

$$
\mathcal{L}=\bar{\psi}\left(i \partial_{t}+\frac{\nabla^{2}}{2 m}\right) \psi,
$$

where $\psi$ is a complex scalar field. Since we need the commutator between density and momentum current, we need to compute the Noether currents for $U(1)$ symmetry and translations. The symmetry transformation is given by

$$
\begin{aligned}
& \psi(t, \boldsymbol{x}) \rightarrow e^{i \alpha} \psi(t, \boldsymbol{x}), \\
& \bar{\psi}(t, \boldsymbol{x}) \rightarrow e^{-i \alpha} \bar{\psi}(t, \boldsymbol{x}) .
\end{aligned}
$$

We promote the parameter $\alpha$ to be a function of space and time and perform a variation of the action with respect to it,

$$
\begin{aligned}
\delta S= & \alpha(t, \boldsymbol{x})\left[\partial_{t}(\bar{\psi}(t, \boldsymbol{x}) \psi(t, \boldsymbol{x}))+\frac{i}{2 m}\left(\nabla^{2} \bar{\psi}(t, \boldsymbol{x}) \psi(t, \boldsymbol{x})\right.\right. \\
& \left.\left.-\bar{\psi}(t, \boldsymbol{x}) \nabla^{2} \psi(t, \boldsymbol{x})\right)\right] .
\end{aligned}
$$

We can now identify the density,

$$
\rho(t, \boldsymbol{x})=\bar{\psi}(t, \boldsymbol{x}) \psi(t, \boldsymbol{x}),
$$


and the particle current density from which we can compute the momentum density,

$$
p_{i}=\frac{i}{2}\left[\nabla_{i} \bar{\psi}(t, \boldsymbol{x}) \psi(t, \boldsymbol{x})-\bar{\psi}(t, \boldsymbol{x}) \nabla_{i} \psi(t, \boldsymbol{x})\right] .
$$

Taking $\{\psi(\boldsymbol{x}), \bar{\psi}(\boldsymbol{y})\}=\delta(\boldsymbol{x}-\boldsymbol{y})$, we can derive Eq. (1). In our analysis of the fracton fluids, we will employ the same procedure by postulating the brackets and justifying them using microscopic models.

\section{MONOPOLE-DIPOLE-MOMENTUM ALGEBRA}

Our analysis will be phrased in the language of multipole algebras constructed in Ref. [57]. The basic ingredient of this construction is the simultaneous conservation of the fracton monopole charge $Q$ and the dipole moment $Q^{i}, i=1, \ldots, d$ ranging over the spatial directions. These are defined to be the spatial integrals of their respective densities:

$$
\begin{aligned}
\frac{d Q}{d t} & =\frac{d}{d t} \int d^{d} x \rho=0, \\
\frac{d Q^{i}}{d t} & =\frac{d}{d t} \int d^{d} x x^{i} \rho=0,
\end{aligned}
$$

where $\rho(t, \boldsymbol{x})$ is the fracton charge density.

A sufficient condition to guarantee these conservation laws is a generalized continuity equation of the form

$$
\partial_{t} \rho+\partial_{i} \partial_{j} J^{i j}=0 .
$$

In addition, we assume that the system is translationally invariant, which implies the conservation of momentum

$$
\frac{d P_{i}}{d t}=\frac{d}{d t} \int d^{d} x p_{i}=0 .
$$

The charges $Q, Q^{i}$, and $P_{i}$ naturally act on the system as generators of the symmetry group. Fields $\Phi$ in a given representation will transform according to $\delta_{\alpha} \Phi=\{\Phi, \alpha Q\}$, $\delta_{\beta} \Phi=\left\{\Phi, \beta_{i} Q^{i}\right\}$, and $\delta_{\gamma} \Phi=\left\{\Phi, \gamma^{i} P_{i}\right\}$, where $\alpha, \beta_{i}$, and $\gamma^{i}$ are constant infinitesimal parameters. For example, these symmetries can be represented by a real scalar field $\phi(t, \boldsymbol{x})$ transforming as

$$
\begin{aligned}
& \delta_{\alpha} \phi(t, \boldsymbol{x})=\alpha, \\
& \delta_{\beta} \phi(t, \boldsymbol{x})=\beta_{i} x^{i}, \\
& \delta_{\gamma} \phi(t, \boldsymbol{x})=\gamma^{i} \partial_{i} \phi(t, \boldsymbol{x}) .
\end{aligned}
$$

Another example is that of a complex scalar field $\psi(t, \boldsymbol{x})$ transforming under a global vector transformation

$$
\psi(t, \boldsymbol{x}) \rightarrow e^{i \boldsymbol{\beta} \cdot \boldsymbol{x}} \psi(t, \boldsymbol{x}),
$$

in addition to the usual $U(1)$ transformation in Eq. (10) and the same momentum transformation as for the real scalar in Eq. (17c). In fact, the real scalar $\phi$ transforms precisely like the phase of the complex scalar field $\psi$. Indeed, the lowenergy limit of a theory of $\psi$ in the spontaneously broken phase, in which the global $U(1)$ and vector symmetries are broken, is described by the corresponding Nambu-Goldstone boson, which is precisely the phase $\phi$ of $\psi$. However, it must be noted that not all theories of $\phi$ with the symmetries (17) necessarily arise in this way.
The monopole transformation, $Q$, is nothing but the constant shift symmetry familiar in the context of NambuGoldstone bosons. The dipole transformation, $Q^{i}$, is just the spatial part of the linear shift symmetry familiar in the context of Galileons [62]. In general, one can consider the so-called polynomial shift symmetry whose charges are generated by polynomials in the spatial coordinates of any finite degree [63]. An example of such a scenario that has been studied previously is the traceless scalar theory [11], which in addition to the conserved charges $Q, Q^{i}$ also conserves the second moment $Q^{(2)}=\int d^{d} x\|\mathbf{x}\|^{2} \rho$.

The form of the monopole-dipole-momentum algebra (MDMA) is as follows,

$$
\begin{aligned}
\{Q, Q\} & =\left\{Q, P_{i}\right\}=\left\{Q, Q^{i}\right\}=0, \\
\left\{P_{i}, Q^{j}\right\} & =\delta_{i}^{j} Q .
\end{aligned}
$$

Notice that momentum and dipole charge are charged with respect to each other. In fact, if we shift the origin of the coordinates system in some direction $(\gamma)$, its dipole moment will change accordingly to $\delta_{\gamma} Q^{i}=\gamma^{i} Q$. On the other hand, it is less intuitive that a dipole transformation with parameter $\boldsymbol{\beta}$ likewise modifies the momentum. Nevertheless, the algebra (19a) dictates that it does it according to $\delta_{\beta} P_{i}=\beta_{i} Q$, which can be written in terms of the densities as follows

$$
\delta_{\beta} p_{i}=\beta_{i} \rho .
$$

In the next sections we will focus on the hydrodynamic description of systems that are invariant under the whole symmetry group. Then, we apply the Poisson bracket formalism to write down hydrodynamic equations for fractons. In particular, we will study two different classes of fracton theories that we dub chiral and nonchiral. However, the structure of hydrodynamic equations appears to be universal. The Poisson brackets (1) do not change, but the final form of the constitutive relations strongly depends on the Hamiltonian. The main distinguishing feature between the nonchiral and the chiral theories is that the former will be assumed to be a time-reversal invariant whereas in the latter case we will allow for the breaking of time-reversal invariance while preserving a combination of time inversions and a $\pi$ rotation.

In our analysis we do not introduce an additional independent charge for the dipole symmetry because the dipole charge is a derived quantity from the ordinary scalar charge. The situation is analogous to the orbital angular momentum or, in conformal theories, the charges associated to scale and conformal transformations. Those are not assigned independent charges but are constructed from the components of the energy-momentum tensor. Their conservation follows from the properties of the energy-momentum tensor, conservation, symmetry, and tracelessness. Similarly, the conservation of the dipole charge follows from the properties of the charge current, which is a total derivative of a symmetric tensor. One might consider the presence of an intrinsic dipole charge with a separate charge density, but we would not pursue this possibility here. 


\section{HYDRODYNAMICS OF NONCHIRAL FRACTONS}

We start with theories preserving time-reversal invariance. First we will do a general analysis of hydrodynamics based on symmetries alone. In the hydrodynamic description we have to postulate the form of the Poisson brackets between conserved charges based on the action they are expected to have as symmetry generators. On the other hand, the dependence of the Hamiltonian on the charges is constrained by the symmetries. Combining these two together, we are able to derive the constitutive relations for the currents in terms of the conserved charge and momenta. We do not make any assumption about the underlying microscopic theory other than the symmetries. Once we derive the hydrodynamic equations and constitutive relations, we proceed to confirm our assumptions about the Poisson brackets and the hydrodynamic equations that follow by computing them in simple models where the symmetries are realized explicitly.

For the present case, we find it convenient to introduce the generalized velocity $V_{i}=\rho^{-1} p_{i}$ which transforms as $\delta_{\beta} V_{i}=$ $\beta_{i}$ [see Eq. (20)] and constrain the Hamiltonian to depend on the momentum only via the $\beta$-invariant combination $\partial_{i} V_{j}$. In fact, notice that a linear shift acts similarly to a Galilean boost, since the generalized velocity is shifted by the "boost" parameter $\beta_{i}$. Therefore we postulate that the low-energy effective Hamiltonian of the system has the following form:

$$
H=\int d^{d} x h\left(\rho, \partial_{i} V_{j}, \partial_{i} \rho\right)+\mathcal{O}\left(\partial^{3}\right) .
$$

Since the independent dynamical variables of the system are $\rho$ and $p_{i}$, the variation of the Hamiltonian will have the form ${ }^{2}$

$$
\delta H=\int d^{d} x\left(\mu \delta \rho+v^{i} \delta p_{i}\right)
$$

where the out-of-equilibrium chemical potentials take the form

$$
\begin{aligned}
v^{i} & =-\frac{1}{\rho} \partial_{j} \frac{\partial h}{\partial\left(\partial_{j} V_{i}\right)}, \\
\mu & =\frac{\partial h}{\partial \rho}-\partial_{i} \frac{\partial h}{\partial\left(\partial_{i} \rho\right)}-\frac{p_{i} v^{i}}{\rho} .
\end{aligned}
$$

In addition, we postulate the same Poisson brackets (1) as for ordinary fluids. In fact, in the next section we shall verify for a certain class of theories with linear shift symmetry that our assumptions are correct. We now derive the equations of motion for the conserved densities using $\partial_{t} S=\{S, H\}$, which are the same as Eq. (3). However, in the present case, $\rho v^{i}$ is a gradient as expected for a fractonic system. Therefore the constitutive relation for the fractonic current reads

$$
J^{i j}=-\frac{\partial h}{\partial\left(\partial_{(j} V_{i)}\right)} .
$$

\footnotetext{
${ }^{2}$ Notice that we are not considering the dipole density $\rho^{i}(t, \mathbf{x})=$ $x^{i} \rho(t, \mathbf{x})$ as a hydrodynamical variable since its conservation follows from the conservation of the charge density $\rho(t, \mathbf{x})$.
}

On the other hand, we can read out the momentum conservation equation the stress tensor

$$
T_{j}^{i}=p \delta_{j}^{i}+v^{i} p_{j}+\frac{\partial h}{\partial\left(\partial_{i} \rho\right)} \partial_{j} \rho+\frac{\partial h}{\partial\left(\partial_{i} V_{k}\right)} \partial_{j} V_{k},
$$

with the pressure $p$ defined as $p=\rho \mu+p_{i} v^{i}-h$.

To be more precise and understand the consequences of this proposal, we consider a time-reversal invariant quadratic Hamiltonian which would capture the dynamics of linear perturbations around the equilibrium state $\rho=\rho_{0}, p_{i}=0$. The most general such Hamiltonian density reads

$$
h=\frac{\mu_{0}}{2 \rho_{0}} \rho^{2}+\frac{1}{2 \rho_{0}} \mu_{1}^{i j} \partial_{i} \rho \partial_{j} \rho+\frac{\rho_{0}}{2} v_{1}^{i j k l} \partial_{i} V_{j} \partial_{k} V_{l},
$$

with the phenomenological susceptibilities being positive definite in order that the Hamiltonian be bounded from below. Then, we fix the background density $\rho=\rho_{0}$ and pick the "frame" $p_{i}=0$. In particular, the out-of-equilibrium chemical potentials $\mu$ and $v^{i}$ are

$$
\begin{gathered}
\mu=\rho_{0}^{-1}\left(\mu_{0} \rho-\mu_{1}^{i j} \partial_{i} \partial_{j} \rho\right), \\
v^{j}=-v_{1}^{i j k l} \partial_{i} \partial_{k} V_{l},
\end{gathered}
$$

which imply the following set of linearized hydrodynamic equations

$$
\begin{gathered}
\partial_{t} \delta \rho-v_{1}^{i j k l} \partial_{i} \partial_{j} \partial_{k} \delta p_{l}=0, \\
\partial_{t} \delta p_{i}+\left(\mu_{0}-\mu_{1}^{j k} \partial_{j} \partial_{k}\right) \partial_{i} \delta \rho=0 .
\end{gathered}
$$

After Fourier transforming the system of equations, it is possible to verify the existence of two modes, One of which is nonpropagating (at $\omega=0)^{3}$ while the propagating mode has the following dispersion relation

$$
\omega^{2}=\mu(q) v_{1}^{i j k l} q_{i} q_{j} q_{k} q_{l},
$$

where $\mu(q)=\mu_{0}+\mu_{1}^{i j} q_{i} q_{j}$. Note that the positivity conditions required of all the susceptibilities appearing in Eq. (26) guarantee that $\mu(q) v_{1}^{i j k l}$ is positive definite and, therefore, that the mode with dispersion (31) will be a stable propagating mode. Also notice that the antisymmetric components $v_{1}^{[i j][k l]}$ are only relevant at the nonlinear level since they do not appear in the linearized equations of motion.

\section{A. Real Scalar field with linear shift symmetry}

In this section we will confirm the Poisson algebra and structure assumed in the hydrodynamic Hamiltonian for the fracton system with linear shift symmetry (17). In particular we shall assume theories with Lagrangian of the form

$$
L=\int d^{d} x \mathcal{L}\left(\partial_{t} \phi, \partial_{i} \partial_{j} \phi\right) .
$$

For simplicity and clarity of presentation, we will consider this restricted functional dependence of the Lagrangian on the derivatives of the scalar field. Although a priori this theory

\footnotetext{
${ }^{3}$ In general, we expect that dissipative terms will modify the dispersion relation of the nonpropagating mode.
} 
does allow for chiral modes, we impose an additional symmetry requirement that they are absent. The generalization to Lagrangians depending on higher-order spatial derivatives of the scalar field is quite straightforward but leads only to a proliferation of terms with no significant added insight. Such a form of the Lagrangian ensures that $\mathcal{L}$ by itself is invariant under the linear shift symmetry and not just its spatial integral $L$. We can allow $\mathcal{L}$ to vary by a total derivative, in which case it can depend on the first spatial derivative $\partial_{i} \phi$ as long as we insist that $\frac{\partial \mathcal{L}}{\partial\left(\partial_{i} \phi\right)}$ is itself the gradient of some expression. Again, it is easy to handle adding this extra functional dependence in Eq. (32), but it does not lead to any added insight, at least insofar as the present analysis is concerned. Thus we choose to restrict to the form (32) (see the Appendix for the more general case).

After taking variations of the Lagrangian, we obtain

$$
\begin{aligned}
\delta L= & -\int d^{d} x\left(\partial_{t} \pi+\partial_{i} \partial_{j} J^{i j}\right) \delta \phi \\
& +\int d^{d} x\left(\partial_{t}(\pi \delta \phi)+\partial_{i}\left(\partial_{j} J^{i j} \delta \phi-J^{i j} \partial_{j} \delta \phi\right)\right),
\end{aligned}
$$

where we have defined

$$
\pi=\frac{\partial \mathcal{L}}{\partial\left(\partial_{t} \phi\right)}, \quad J^{i j}=-\frac{\partial \mathcal{L}}{\partial\left(\partial_{i} \partial_{j} \phi\right)} .
$$

For simplicity, we assume that the time derivative of the field can be obtained from $\pi$ by inverting with some functional $F$

$$
\partial_{t} \phi=F\left(\pi, \partial_{i} \partial_{j} \phi\right) \text {. }
$$

In the next section we will consider the example of a theory where this is not possible. From the variation of the action we can simultaneously read the equations of motion and the Noether currents associated with the constant and linear shift. In fact, the equation of motion reads

$$
\partial_{t} \pi+\partial_{i} \partial_{j} J^{i j}=0,
$$

which can be identified with the monopole charge conservation with $\rho=\pi$. In addition, the dipole density defined as $\rho^{i}=x^{i} \pi$ is also conserved,

$$
\partial_{t} \rho^{k}+\partial_{i} j^{i k}=0
$$

where

$$
j^{i k}=x^{k} \partial_{j} J^{i j}-J^{i k} .
$$

The system is also translationally invariant in time and space, which implies the conservation of energy and momentum. The energy density and flux are

$$
\begin{gathered}
h=\pi \partial_{t} \phi-\mathcal{L}\left(F, \partial_{i} \partial_{j} \phi\right), \\
T^{i}{ }_{t}=\left(\partial_{j} J^{i j}\right) \partial_{t} \phi-J^{i j} \partial_{j} \partial_{t} \phi,
\end{gathered}
$$

while the momentum density and stress tensor are

$$
\begin{aligned}
p_{i} & =T^{t}{ }_{i}=-\pi \partial_{i} \phi, \\
T^{j}{ }_{i} & =-\partial_{k} J^{j k} \partial_{i} \phi+J^{j k} \partial_{k} \partial_{i} \phi+\delta_{i}^{j} \mathcal{L}\left(F, \partial_{i} \partial_{j} \phi\right) .
\end{aligned}
$$

They satisfy the usual conservation equations

$$
\begin{aligned}
\partial_{t} h+\partial_{i} T^{i}{ }_{t} & =0, \\
\partial_{t} p_{i}+\partial_{j} T^{j}{ }_{i} & =0 .
\end{aligned}
$$

The definition of the momentum and charge densities for this class of systems automatically satisfies the transformation rule (20), and the Hamiltonian density (39a) can be written as a functional of $\rho$ and $\partial_{i} V_{j}$,

$$
h \equiv h\left(\rho, \partial_{i} V_{j}\right) \text {, }
$$

as expected. Finally, for this class of theories, it is not hard to verify that the canonical bracket

$$
\{\phi(\boldsymbol{x}), \pi(\boldsymbol{y})\}=\delta(\boldsymbol{x}-\boldsymbol{y})
$$

implies the usual algebra (1) used above to derive the hydrodynamic theory.

\section{B. Complex scalar field with linear shift symmetry}

Imposing the global vector symmetry (18) on a theory of a complex scalar field $\psi$ forbids an ordinary kinetic term that is quadratic in $\psi$ and contains some number of spatial derivatives. Instead, the theory of this kind that has been considered previously in $[59,60]$ reads

$$
\begin{aligned}
\mathcal{L}= & \left|\partial_{t} \psi\right|^{2}-m^{2}|\psi|^{2}-\frac{\lambda}{4}\left(|\psi|^{2}\right)^{2}-c_{1} \partial_{i}|\psi|^{2} \partial_{i}|\psi|^{2} \\
& -c_{2}\left|\chi_{i j}\right|^{2}-c_{3}\left[\left(\psi^{*}\right)^{2} \chi+\text { H.c. }\right],
\end{aligned}
$$

where H.c. is the Hermitian conjugate,

$$
\chi_{i j} \equiv \psi \partial_{i} \partial_{j} \psi-\partial_{i} \psi \partial_{j} \psi,
$$

and $\chi=\chi_{i i}$ is its trace. The canonical momenta of this system are $\pi_{\psi}=\partial_{t} \bar{\psi}$ and $\pi_{\bar{\psi}}=\partial_{t} \psi$. For such theory the $U(1)$ Noether's charge $\rho$ and current $J^{i} \equiv \partial_{j} J^{i j}$ densities are

$$
\begin{aligned}
\rho & =i\left(\bar{\psi} \partial_{t} \psi-\text { H.c. }\right), \\
J_{i j} & =i c_{2}\left(\bar{\psi}^{2} \chi_{i j}-\text { H.c. }\right) .
\end{aligned}
$$

On the other hand, the translational invariance of the system implies conservation of momentum. In particular, the momentum and stress densities are

$$
\begin{aligned}
p_{i}= & -\left(\partial_{i} \bar{\psi} \pi_{\psi}+\text { H.c. }\right), \\
T^{j}{ }_{i}= & \mathcal{L} \delta_{i}^{j}+2 c_{1} \partial_{j}|\psi|^{2} \partial_{i}|\psi|^{2} \\
& +c_{2}\left(\bar{\chi}_{j k}\left(\chi_{k i}-2 \partial_{k} \psi \partial_{i} \psi\right)-\frac{1}{2} \partial_{k} \bar{\chi}_{j k} \partial_{i} \psi^{2}+\text { H.c. }\right) \\
& +c_{3}\left(\bar{\psi}^{2}\left(\chi_{i j}-2 \partial_{j} \psi \partial_{i} \psi\right)-2|\psi|^{2} \partial_{j} \bar{\psi} \partial_{i} \psi+\text { H.c. }\right) .
\end{aligned}
$$

In addition, the system's Hamiltonian and energy current read

$$
\begin{aligned}
h= & 2\left|\partial_{t} \psi\right|^{2}-\mathcal{L} \\
T^{i}{ }_{t}= & -2 c_{1} \partial_{t}|\psi|^{2} \partial_{i}|\psi|^{2}-c_{2}\left(\bar{\chi}_{i j}\left(\psi \partial_{j} \pi_{\bar{\psi}}-3 \partial_{j} \psi \pi_{\bar{\psi}}\right)\right. \\
& \left.-\partial_{j} \bar{\chi}_{i j} \psi \pi_{\bar{\psi}}+\text { H.c. }\right)-c_{3}\left(\bar{\psi}^{2}\left(\psi \partial_{i} \pi_{\bar{\psi}}-3 \partial_{i} \psi \pi_{\bar{\psi}}\right)\right. \\
& \left.-2|\psi|^{2} \partial_{i} \bar{\psi} \pi_{\bar{\psi}}+\text { H.c. }\right) .
\end{aligned}
$$

Using the equations of motion for this theory, one can verify that conservation Eqs. (15) and (41) are satisfied. Furthermore, the same canonical brackets as in Eq. (43) for $\psi, \bar{\psi}$ and their conjugate momenta imply the usual algebra between the conserved densities Eq. (1). 
In the broken phase, in which $|\psi|$ picks up a vacuum expectation value $\langle|\psi|\rangle$ at energies well below the mass scale, $E \ll$ $m$, the theory just contains the Nambu-Goldstone mode, which is the phase $\phi$ of $\psi$. After rescaling time and space by a factor of $\langle|\psi|\rangle$, the Lagrangian is simply given by a free massless $z=2$ real scalar field theory: $\mathcal{L}=\frac{1}{2}\left(\partial_{t} \phi\right)^{2}-\frac{1}{4} c_{2}\left(\partial_{i} \partial_{j} \phi\right)^{2}$, which is a simple special case of the Lagrangian (32) in the previous example.

\section{HYDRODYNAMICS OF CHIRAL FRACTONS}

In the cases of physical interest, fractons may not only be restricted to move in a subdimensional space but the direction of motion may be determined as well. An example of this are edge dislocations under an applied stress whose direction of motion is determined by the Burgers vector $\boldsymbol{b}$ and the orientation of the defect. Dual elastic fields couple to the disclination density $\rho$ and current tensor $J^{i j}$ with a conservation Eq. (15) [21]. In this system, the monopole charge $Q$ and the dipole moment $Q^{i}$ can be identified with the disclination and dislocation charge, respectively. In a two-dimensional lattice, the contribution to the current tensor of a dislocation moving with velocity $\boldsymbol{v}$ takes the form [21]

$$
J^{i j} \propto \epsilon^{(i k} b_{k} v^{j)} .
$$

In the absence of vacancies, the current tensor is traceless $\mathrm{J}^{i}{ }_{i}=0[42,64]$, forcing the velocity to be parallel to the Burgers vector $v^{i}=b^{i} v_{b}$.

Let us introduce the unit vector $\hat{\boldsymbol{b}}=\boldsymbol{b} /\|\boldsymbol{b}\|$ and define the coordinates parallel and transverse to the Burgers vector, $b=$ $\hat{b}_{i} x^{i}, s=\epsilon^{i j} x_{i} \hat{b}_{j}$, as well as the corresponding derivatives

$$
\partial_{b} \equiv \hat{b}^{i} \partial_{i}, \quad \partial_{s} \equiv \epsilon^{i j} \hat{b}_{j} \partial_{i} .
$$

With these conventions and assuming no vacancies, the current is

$$
J^{b b}=J^{s s}=0, \quad J^{b s}=J^{s b} \propto \frac{1}{2} v_{b} .
$$

We expect this to be a general form for the constitutive relation of the current. However, there are some subtleties regarding the realization of symmetries, as the breaking of rotational invariance also allows for subsystem symmetries. In this case we will follow a different path than that for the nonchiral models. We will start by studying a simple model with the right symmetry realization and use it to derive the constitutive relations for the currents and hydrodynamic equations. Once expressed in terms of conserved charges, one could in principle forget about the microscopic origin and postulate the result as a hydrodynamic description for this class of models. However, it is possible that the constitutive relations derived in this form are not the most general, and although we expect the general structure to be correct, some of the values of the coefficients might be allowed to change.

This structure can be captured by a simple model with a scalar field. The action reads

$$
S=\int d^{3} x \mathcal{L}=\frac{1}{2} \int d^{3} x \partial_{s} \phi\left(\partial_{t} \phi+\partial_{b} \phi\right) .
$$

The equation of motion is

$$
\partial_{t} \partial_{s} \phi+\partial_{b} \partial_{s} \phi=0 .
$$

This takes the form of the continuity Eq. (15) if we identify

$$
\rho \propto \partial_{s} \phi, \quad J^{s s}=J^{b b}=0, \quad J^{b s} \propto \frac{1}{2} \phi,
$$

where the proportionality constants are the same for both $\rho$ and $J^{b s}$. The energy, momentum, and charge densities in the Lagrangian formalism are

$$
\begin{aligned}
e & =T_{t}^{t}=\frac{\delta S}{\delta \partial_{t} \phi} \partial_{t} \phi-\mathcal{L}=-\frac{1}{2} \partial_{s} \phi \partial_{b} \phi, \\
k_{i} & =T_{i}^{t}=-\frac{\delta S}{\delta \partial_{t} \phi} \partial_{i} \phi=-\frac{1}{2} \partial_{s} \phi \partial_{i} \phi, \\
n & =\frac{\delta S}{\delta \partial_{t} \phi}=\frac{1}{2} \partial_{s} \phi .
\end{aligned}
$$

Therefore, the model captures the structure of Eq. (51) for dislocations of a fixed orientation. Dislocations of different orientations would be described by discrete spatial rotations of the action above.

This model actually has a much larger symmetry than the one implied by the monopole, dipole, and second moment conservation. The action transforms by a total derivative under the transformations

$$
\phi \rightarrow \phi+f(s)+g(t, b),
$$

which are also symmetries of the equation of motion. This implies that there is an infinite set of conserved charges

$$
Q(f, g)=\int d^{2} x(f(s)+g(t, b)) n \text {. }
$$

This model will also have blueuced conformal symmetry as those studied in Ref. [65]. The $s$-independent transformation leaves $q$ and $k_{s}$ invariant but changes the momentum $k_{b}$ as follows:

$$
\delta k_{b}=-n \partial_{b} g(t, b) .
$$

Moving on to the Hamiltonian formalism, the canonical momentum conjugate to the scalar field is

$$
\pi=\frac{\delta S}{\delta \partial_{t} \phi}=\frac{1}{2} \partial_{s} \phi
$$

This relation actually imposes a constraint on phase space, which has to be treated with some care. We will work in the extended phase space parametrized by canonically conjugate variables $(\phi, \pi)$ and impose the constraint after evaluating the Poisson brackets as is standard in these cases. We will use the symbol $=$ for results in the extended space and $\approx$ for results after imposing the constraint.

In the Hamiltonian formalism, the corresponding Hamiltonian and momentum densities differ from Eq. (55) by terms proportional to the constraint that vanish when evaluated on the physical constrained phase space,

$$
\begin{gathered}
h=e+\lambda_{t}\left(\pi-\frac{1}{2} \partial_{s} \phi\right), \\
p_{i}=k_{i}+\lambda_{i}\left(\pi-\frac{1}{2} \partial_{s} \phi\right), \\
\rho=n+\lambda\left(\pi-\frac{1}{2} \partial_{s} \phi\right) .
\end{gathered}
$$


The Lagrange multipliers are fixed by the condition that the charges act as the generators of time and space translations and shifts of the scalar on the physical space. The Hamiltonian, momentum and charge are

$$
H=\int d^{2} x h, \quad P_{i}=\int d^{2} x p_{i}, Q=\int d^{2} x \rho .
$$

Then,

$$
\{\phi, H\} \approx \lambda_{t}, \quad\left\{\phi, P_{i}\right\} \approx \lambda_{i}, \quad\{\phi, Q\} \approx \lambda,
$$

which fixes $\lambda_{t}=\partial_{t} \phi, \lambda_{i}=-\partial_{i} \phi$, and $\lambda=1$. In this case the densities $h, p_{i}$, and $\rho$ in the extended phase space take the usual form for an ordinary scalar, so they lead to the same Poisson brackets and hydrodynamic equations in the extended space. Imposing the constraint fixes the density and the momentum in the direction transverse to the Burgers vector in terms of the same quantity,

$$
\rho \approx \frac{1}{2} \partial_{s} \phi, \quad p_{s} \approx-\frac{1}{2}\left(\partial_{s} \phi\right)^{2} .
$$

This can be interpreted as an equation of state for the fractonic fluid relating the momentum and the disclination density

$$
p_{s}=-2 \rho^{2} \text {. }
$$

In this case, the hydrodynamic equation for $p_{s}$ becomes a constraint.

Solving for the spatial derivatives of $\phi$ and the conjugate momentum, the Hamiltonian is

$$
\begin{aligned}
h= & p_{b}+\frac{\left(\lambda_{t}-\lambda_{b}\right)}{4 \lambda^{2}}\left(\lambda_{s}+4 \lambda \rho\right. \\
& \left.+\operatorname{sgn}\left(\lambda_{s}\right) \sqrt{\lambda_{s}^{2}+8 \lambda\left(\lambda_{s} \rho-\lambda p_{s}\right)}\right) .
\end{aligned}
$$

We will take this as the Hamiltonian for a hydrodynamic theory. For $\lambda_{s} \neq 0$ one finds that, imposing the conditions,

$$
\frac{\partial h}{\partial \lambda_{\mu}}=0, \quad \frac{\partial h}{\partial \lambda}=0,
$$

fixes

$$
p_{s}=-2 \rho^{2}, \quad \lambda_{s}=-2 \lambda \rho .
$$

These are the same conditions we have in the microscopic model.

Then, identifying

$$
\mu=\frac{\partial h}{\partial \rho}, \quad v^{a}=\frac{\partial h}{\partial p_{a}},
$$

subject to the previous conditions, one gets

$$
\lambda_{t}=\lambda_{b}+\frac{\lambda}{2} \mu, \quad v^{s}=\frac{\mu}{4 \rho}, \quad v^{b}=1 .
$$

And, evaluating the Hamiltonian,

$$
h=p_{b} .
$$

Thus the energy equals the momentum in the direction of the Burgers vector. Note that we should impose the conditions after deriving the equations.

The hydrodynamic equation for $p_{s}$ becomes proportional to the equation for $\rho$ but is missing a term depending on the chemical potential. We are left with two independent equations and a constraint on $\mu$ :

$$
\begin{aligned}
& \partial_{t} \rho+\partial_{b} \rho=0, \quad \partial_{s} \mu=0, \\
& \partial_{t} p_{b}+\partial_{b}\left(p_{b}+\frac{1}{2} \mu \rho\right)+\partial_{s}\left(p_{b} \frac{\mu}{4 \rho}\right)=0 .
\end{aligned}
$$

The equation for $\rho$ coincides with the equation of motion in the microscopic model. Note that $\mu$ is not a function of the density or the momenta; rather, its role is to ensure that the symmetry (58) is realized in the hydrodynamic equations, as we will see presently.

Expanding to linear order around constant background values $\left(\rho_{0}, \mu_{0}\right)$ for the density and chemical potential, we get the set of equations

$$
\begin{aligned}
& \partial_{t} \delta \rho+\partial_{b} \delta \rho=0, \quad \partial_{s} \delta \mu=0 \\
& \partial_{t} \delta p_{b}+\partial_{b}\left(\delta p_{b}+\frac{1}{2} \mu_{0} \delta \rho+\frac{1}{2} \rho_{0} \delta \mu\right)+\frac{\mu_{0}}{4 \rho_{0}} \partial_{s} \delta p_{b}=0 .
\end{aligned}
$$

The solutions for the density and chemical potential should take the form

$$
\delta \rho=\delta \bar{\rho}(b-t, s)+\rho_{0} \alpha(b-t), \quad \delta \mu=\delta \mu(t, b),
$$

where $\partial_{s} \delta \bar{\rho} \neq 0$. We have to distinguish between the contributions to the chemical potential that depend on $b-t$ and the remainder. We will split the chemical potential as follows:

$$
\delta \mu=\mu_{0} \beta(b-t)+\left(\partial_{t}+\partial_{b}\right) \varphi(t, b) .
$$

In the momentum we can separate a part that will give a contribution independent of the coordinate $s$ to the conservation equation from another part that can in principle have an arbitrary dependence on $s$ :

$$
\delta p_{b}=\delta \bar{p}_{b}(t, b, s)+\rho_{0}\left[\psi(t, b)+\rho_{0}\left(s-s_{0}\right) \gamma(b-t)\right] .
$$

We are left with the equations

$$
\begin{aligned}
& \left(\partial_{t}+\partial_{b}\right) \delta \bar{p}_{b}+\frac{\mu_{0}}{2} \partial_{b} \delta \bar{\rho}+\frac{\mu_{0}}{4 \rho_{0}} \partial_{s} \delta \bar{p}_{b}=0, \\
& \rho_{0}\left(\partial_{t}+\partial_{b}\right)\left(\psi+\frac{1}{2} \partial_{b} \varphi\right)+\frac{\mu_{0} \rho_{0}}{2}\left(\partial_{b} \alpha+\partial_{b} \beta+\frac{1}{2} \gamma\right)=0 .
\end{aligned}
$$

The solutions are

$$
\begin{aligned}
\gamma & =-2 \partial_{b} \alpha-2 \partial_{b} \beta, \quad \psi=-\frac{1}{2} \partial_{b} \varphi, \\
\delta \bar{\rho} & =\partial_{s} \delta \bar{J}, \quad \delta \bar{J}=\delta \bar{J}(b-t, s), \\
\delta \bar{p}_{b} & =-2 \rho_{0} \partial_{b} \delta \bar{J}+\delta p_{b}^{h} .
\end{aligned}
$$

The homogeneous term $\delta p_{b}^{h}$ can be expanded in plane waves propagating with speed unity in the $b$ direction and speed $c_{s}=\mu_{0} /\left(4 \rho_{0}\right)=v_{0}^{s}$ in the $s$ direction,

$$
\delta p_{b}^{h}=\int \frac{d^{2} q}{(2 \pi)^{2}} \tilde{p}_{b}(q) e^{i q_{s}\left(s-c_{s} t\right)+i q_{b}(b-t)} .
$$

We can identify $\delta \bar{J}$ as part of the current, with an additional contribution parametrized by $\alpha$,

$$
\delta J=\delta \bar{J}+\rho_{0}\left(s-s_{0}\right) \alpha .
$$


The solution for the chemical potential (74) confirms its part in the realization of the symmetries. Compared with the symmetry transformation of $p_{b}(58)$ generated by $g(t, b)$ (the constraint has already been imposed at this point and so $p_{b}$ and $k_{b}$ are identified), we see that $\varphi$ and $\psi$ correspond to said transformation with a condition $\left(\partial_{t}+\partial_{b}\right) g(t, b) \neq 0$.

Thus, there are three types of modes propagating momentum $p_{b}$ : Those associated with the current $\delta J$ and those appearing in the homogeneous contribution $\delta p_{b}^{h}$ and the mode $\beta$. This last mode can be absorbed in the homogeneous part when $\mu_{0} \rightarrow 0$ so that $c_{s} \rightarrow 0$.

\section{DISCUSSION}

We have derived hydrodynamic equations and constitutive relations for ideal fluids with a dipole symmetry. Our derivation utilizes representative microscopic scalar models and the Poisson brackets between densities of conserved charges, following the classical approach pioneered by Landau. We have considered three types of models: Two that we dub "nonchiral fracton and a third, "chiral fracton," which contains a vector that can be identified with the Burgers vector in the fracton description of disclinations and dislocations. In the former we introduce two spatial derivatives per field so that there is either a shift or U(1) symmetry linear in the spatial coordinates, and in the case of the real field, allow terms with an arbitrary number of fields. In the chiral fracton model, there is just one derivative per field and is truncated to quadratic order. All models produce the same hydrodynamic equations when written in terms of the densities but with distinct constitutive relations.

In all cases the particle number current is a total derivative either by construction in the nonchiral models (23a) or on-shell in the chiral fracton model (77) in such a way that the dipole moment of the charge is conserved in both. We have studied the spectrum of fluctuations around a state with fixed monopole density. In the nonchiral case there are modes with a dispersion relation $\omega^{2} \sim q^{4}(31)$, where $q^{4}$ stands for a general quartic polynomial of the momenta with coefficients determined by derivatives of the energy density with respect to the gradient of the generalized velocity $V_{i}=\rho^{-1} p_{i}$. In the chiral fracton model, there are chiral modes propagating in the direction of the Burgers vector as well as a mode that also propagates energy and momentum in the transverse direction (78) with a velocity propotional to the chemical potential.

In Ref. [34] charge diffusion was studied for a model sitting within the nonchiral systems we considered. However, they did not consider momentum conservation. A complete finite-temperature hydrodynamic theory should account for both charge and momentum conservation, as we have done in this paper, but the theory should also include dissipative effects, which is an aspect that we leave for future studies. Nonetheless, a peculiarity of systems with MDMA symmetry is the presence of a nonballistic propagating mode and a diffusive one with dispersion relation $\omega \sim-i q^{4}$ [34], contrary to ordinary hydrodynamics where sound waves are ballistic and have a quadratic dispersion relation for diffusive modes.

On the other hand, defect dynamics have important ramifications in crystals, amorphous solids, liquid crystals, and active matter [66,67]. Although the continuous description of defects as a gauge theory has a long history, a symmetry-based construction of defect hydrodynamics beyond vortices is still lacking and various phenomenological equations have been proposed for specific systems (see e.g., Refs. [68-75]). In order to fill this gap, we have proposed a Poisson bracket derivation of hydrodynamic equations given two main ingredients that are important in modeling macroscopic systems of defects. The first ingredient is invariance under MDMA symmetry; the second ingredient is the existence of a Burgers vector. These requirements can be viewed as basic guiding principles for hydrodynamic theories of defects as can be justified using the dual fracton picture. A model displaying the MDMA symmetry and a Burgers vector is the chiral fracton theory of a scalar field. Interpreting the model as a description of lattice defects, the chiral propagation of the disclination density has a simple visualization. In any symmetric region of finite area, there is a finite number of disclinations determined by the integration of the constant charge density in the region. The dipole moment, on the other hand, vanishes, indicating a net zero charge of dislocations. However, as the dislocation density is not identically zero, there is a nonzero amount of dislocation pairs. While immobile in isolation, disclinations can move by absorbing or emitting dislocation pairs [72,7679], in which case their motion is along the Burgers vector of the dislocations. This matches nicely with the behavior of the solutions we obtain in the chiral fracton model. Thus the results of this paper provide basic building blocks to construct more realistic models and ultimately study transport properties of defects.

\section{ACKNOWLEDGMENTS}

K.T.G. and F.P.-B. acknowledge financial support by the Deutsche Forschungsgemeinschaft (DFG, German Research Foundation) under Germany's Excellence Strategy through the Würzburg-Dresden Cluster of Excellence on Complexity and Topology in Quantum Matter - ct.qmat (EXC 2147, project-id 390858490). K.T.G. also acknowledges the support of the Hallwachs-Röntgen Postdoc Program of ct.qmat. C.H. has been partially supported by the Spanish Ministerio de Ciencia, Innovación y Universidades through Grant PGC2018-096894-B-100. P.S. was supported by the Deutsche Forschungsgemeinschaft through the Leibniz Program, the cluster of excellence ct.qmat (EXC 2147, project-id 39085490), and the Narodowe Centrum Nauki (Polish National Science Centre) Sonata Bis Grant 2019/34/E/ST3/00405.

\section{APPENDIX: MORE GENERAL LAGRANGIANS}

Suppose we allow for the more general dependence of the Lagrangian density (32) of the form

$$
L=\int d^{d} x \mathcal{L}\left(\partial_{t} \phi, \partial_{i} \phi, \partial_{i} \partial_{j} \phi, \ldots\right),
$$

where ... stands for higher spatial derivatives of $\phi$. Let us define the variational quantities

$$
\pi=\frac{\partial \mathcal{L}}{\partial\left(\partial_{t} \phi\right)},
$$




$$
\pi^{i_{1} \cdots i_{n}}=(-1)^{n-1} \frac{\partial \mathcal{L}}{\partial\left(\partial_{i_{1}} \cdots \partial_{i_{n}} \phi\right)}, \quad n \geqslant 1 .
$$

For simplicity we introduce the notation $\overrightarrow{1}_{n}=\left(i_{1}, \ldots, i_{n}\right)$ to be an $n$ tuple of indices and

$$
\pi^{\overrightarrow{1}_{n}}=\pi^{i_{1} \cdots i_{n}}, \quad \partial_{\overrightarrow{1}_{n}}^{n}=\partial_{i_{1}} \cdots \partial_{i_{n}} .
$$

Indices can be concatenated, $\pi^{i \overrightarrow{\mathrm{J}}_{n}}=\pi^{i j_{1} \cdots j_{n}}$, and similarly for the partial derivatives, $\partial_{i \vec{l}_{n}}^{n+1}=\partial_{i} \partial_{j_{1}} \cdots \partial_{j_{n}}$.

Then, as discussed earlier, for this theory to be invariant under the linear shift, we require that

$$
\pi^{i}=\frac{\partial \mathcal{L}}{\partial\left(\partial_{i} \phi\right)}=\partial^{i} \chi,
$$

for some functional $\chi$ of $\phi$ and its derivatives.

With this in place, the only difference between this case and the simpler case considered in the main text is that some expressions become more complicated. The density $\rho$ is still defined to be $\rho=\pi$. However, the expression for $J^{i j}$ in Eq. (34) is modified to

$$
J^{i j}=\chi \delta^{i j}+\sum_{n \geqslant 0} \partial_{\vec{k}_{n}}^{n} \pi^{i j \vec{k}_{n}}
$$

This reduces to the simpler case studied in the main text when we set $\chi=0$ and $\pi^{\overrightarrow{1}_{n}}=0$ for $n \geqslant 3$.

The Hamiltonian and momentum densities are unchanged,

$$
\begin{aligned}
h & =\pi \partial_{t} \phi-\mathcal{L}, \\
p_{i} & =-\pi \partial_{i} \phi,
\end{aligned}
$$

but the corresponding spatial currents (39b) and (40b) are modified to

$$
\begin{aligned}
& T^{i}{ }_{t}=\left(\partial_{j} J^{i j}\right) \partial_{t} \phi+\left(\chi \delta^{i j}-J^{i j}\right) \partial_{j} \partial_{t} \phi \\
& +\sum_{m \geqslant 2} \sum_{n \geqslant 0}(-1)^{m} \partial_{\vec{k}_{n}}^{n} \pi^{i \overrightarrow{\mathrm{J}}_{m} \vec{k}_{n}} \partial_{\overrightarrow{\mathrm{J}}_{m}} \partial_{t} \phi, \\
& T^{j}{ }_{i}=-\left(\partial_{k} J^{j k}\right) \partial_{i} \phi+\left(J^{j k}-\chi \delta^{j k}\right) \partial_{k} \partial_{i} \phi+\delta_{i}^{j} \mathcal{L} \\
& -\sum_{m \geqslant 2} \sum_{n \geqslant 0}(-1)^{m} \partial_{\vec{k}_{n}}^{n} \pi^{j \vec{\ell}_{m} \vec{k}_{n}} \partial_{\vec{\ell}_{m}}^{m} \partial_{i} \phi \text {. }
\end{aligned}
$$

The equation of motion (36), continuity Eqs. (37), and current relations (38) associated with the constant and linear shift symmetries and the energy-momentum conservation Eqs. (41) associated with time- and space-translation invariance all still hold in precisely the same form.
[1] R. M. Nandkishore and M. Hermele, Fractons, Annu. Rev. Condens. Matter Phys. 10, 295 (2019).

[2] M. Pretko, X. Chen, and Y. You, Fracton phases of matter, Int. J. Mod. Phys. A 35, 2030003 (2020).

[3] C. Chamon, Quantum Glassiness in Strongly Correlated Clean Systems: An Example of Topological Overprotection, Phys. Rev. Lett. 94, 040402 (2005).

[4] J. Haah, Local stabilizer codes in three dimensions without string logical operators, Phys. Rev. A 83, 042330 (2011).

[5] S. Bravyi, B. Leemhuis, and B. M. Terhal, Topological order in an exactly solvable 3d spin model, Ann. Phys. 326, 839 (2011).

[6] S. Vijay, J. Haah, and L. Fu, Fracton topological order, generalized lattice gauge theory and duality, Phys. Rev. B 94, 235157 (2016).

[7] D. J. Williamson, Fractal symmetries: Ungauging the cubic code, Phys. Rev. B 94, 155128 (2016).

[8] C. Xu, Gapless bosonic excitation without symmetry breaking: An algebraic spin liquid with soft gravitons, Phys. Rev. B 74, 224433 (2006).

[9] C. Xu and P. Hořava, Emergent gravity at a lifshitz point from a bose liquid on the lattice, Phys. Rev. D 81, 104033 (2010).

[10] M. Pretko, Subdimensional particle structure of higher rank $u$ (1) spin liquids, Phys. Rev. B 95, 115139 (2017).

[11] M. Pretko, Generalized electromagnetism of subdimensional particles: A spin liquid story, Phys. Rev. B 96, 035119 (2017).

[12] Y. You, Z. Bi, and M. Pretko, Emergent fractons and algebraic quantum liquid from plaquette melting transitions, Phys. Rev. Research 2, 013162 (2020).

[13] S. Pai, M. Pretko, and R. M. Nandkishore, Localization in fractonic random circuits, Phys. Rev. X 9, 021003 (2019); 9, 049901(E) (2019).

[14] J. Feldmeier, P. Sala, G. De Tomasi, F. Pollmann, and M. Knap, Anomalous Diffusion in Dipole- and Higher-
Moment-Conserving Systems, Phys. Rev. Lett. 125, 245303 (2020).

[15] A. Morningstar, V. Khemani, and D. A. Huse, Kinetically constrained freezing transition in a dipole-conserving system, Phys. Rev. B 101, 214205 (2020).

[16] J. Iaconis, A. Lucas, and R. Nandkishore, Multipole conservation laws and subdiffusion in any dimension, Phys. Rev. E 103 022142 (2021).

[17] S. Moudgalya, A. Prem, D. A. Huse, and A. Chan, Spectral statistics in constrained many-body quantum chaotic systems, Phys. Rev. Research 3, 023176 (2021).

[18] M. Pretko and L. Radzihovsky, Symmetry-Enriched Fracton Phases from Supersolid Duality, Phys. Rev. Lett. 121, 235301 (2018).

[19] A. Gromov, Chiral Topological Elasticity and Fracton Order, Phys. Rev. Lett. 122, 076403 (2019).

[20] A. Kumar and A. C. Potter, Symmetry-enforced fractonicity and two-dimensional quantum crystal melting, Phys. Rev. B 100, 045119 (2019).

[21] M. Pretko and L. Radzihovsky, Fracton-Elasticity Duality, Phys. Rev. Lett. 120, 195301 (2018).

[22] M. Pretko, Z. Zhai, and L. Radzihovsky, Crystal-to-fracton tensor gauge theory dualities, Phys. Rev. B 100, 134113 (2019).

[23] Z. Zhai and L. Radzihovsky, Two-dimensional melting via sinegordon duality, Phys. Rev. B 100, 094105 (2019).

[24] A. Gromov and P. Surówka, On duality between Cosserat elasticity and fractons, SciPost Phys. 8, 065 (2020).

[25] D. X. Nguyen, A. Gromov, and S. Moroz, Fracton-elasticity duality of two-dimensional superfluid vortex crystals: Defect interactions and quantum melting, SciPost Phys. 9, 076 (2020).

[26] M. Fruchart and V. Vitelli, Symmetries and Dualities in the Theory of Elasticity, Phys. Rev. Lett. 124, 248001 (2020). 
[27] N. Manoj, R. Moessner, and V. B. Shenoy, Fractonic View of Folding and Tearing Paper: Elasticity of Plates Is Dual to a Gauge Theory with Vector Charges, Phys. Rev. Lett. 127, 067601 (2021).

[28] P. Surówka, Dual gauge theory formulation of planar quasicrystal elasticity and fractons, Phys. Rev. B 103, L201119 (2021).

[29] A. Paramekanti, L. Balents, and M. P. A. Fisher, Ring exchange, the exciton bose liquid, and bosonization in two dimensions, Phys. Rev. B 66, 054526 (2002).

[30] A. W. Sandvik, S. Daul, R. R. P. Singh, and D. J. Scalapino, Striped Phase in a Quantum $x y$ Model with Ring Exchange, Phys. Rev. Lett. 89, 247201 (2002).

[31] V. Rousseau, G. G. Batrouni, and R. T. Scalettar, Phase Separation in the Two-Dimensional Bosonic Hubbard Model with Ring Exchange, Phys. Rev. Lett. 93, 110404 (2004).

[32] J.-K. Yuan, S. A. Chen, and P. Ye, Fractonic superfluids, Phys. Rev. Research 2, 023267 (2020).

[33] S. A. Chen, J.-K. Yuan, and P. Ye, Fractonic superfluids. ii. Condensing subdimensional particles, Phys. Rev. Research 3, 013226 (2021).

[34] A. Gromov, A. Lucas, and R. M. Nandkishore, Fracton hydrodynamics, Phys. Rev. Research 2, 033124 (2020).

[35] D. Aasen, D. Bulmash, A. Prem, K. Slagle, and D. J. Williamson, Topological defect networks for fractons of all types, Phys. Rev. Research 2, 043165 (2020).

[36] E. B. Sonin, Dynamics of Quantised Vortices in Superfluids (Cambridge University Press, Cambridge, 2016).

[37] Z. F. Ezawa, Quantum Hall Effects (World Scientific, Singapore, 2013).

[38] A. M. Turner, V. Vitelli, and D. R. Nelson, Vortices on curved surfaces, Rev. Mod. Phys. 82, 1301 (2010).

[39] K. Bertoldi, V. Vitelli, J. Christensen, and M. van Hecke, Flexible mechanical metamaterials, Nat. Rev. Mater. 2, 17066 (2017).

[40] H. Kleinert, Double gauge theory of stresses and defects, Phys. Lett. A 97, 51 (1983).

[41] J. Zaanen, Z. Nussinov, and S. I. Mukhin, Duality in $2+$ 1D quantum elasticity: Superconductivity and quantum nematic order, Ann. Phys. 310, 181 (2004).

[42] V. Cvetkovic, Z. Nussinov, and J. Zaanen, Topological kinematic constraints: Dislocations and the glide principle, Philos. Mag. 86, 2995 (2006).

[43] A. J. Beekman, J. Nissinen, K. Wu, K. Liu, R.-J. Slager, Z. Nussinov, V. Cvetkovic, and J. Zaanen, Dual gauge field theory of quantum liquid crystals in two dimensions, Phys. Rep. 683, 1 (2017).

[44] P. Wiegmann and A. G. Abanov, Anomalous Hydrodynamics of Two-Dimensional Vortex Fluids, Phys. Rev. Lett. 113, 034501 (2014).

[45] H. Watanabe and H. Murayama, Redundancies in NambuGoldstone Bosons, Phys. Rev. Lett. 110, 181601 (2013).

[46] A. Lucas and P. Surówka, Sound-induced vortex interactions in a zero-temperature two-dimensional superfluid, Phys. Rev. A 90, 053617 (2014).

[47] X. Yu and A. S. Bradley, Emergent Non-Eulerian Hydrodynamics of Quantum Vortices in Two Dimensions, Phys. Rev. Lett. 119, 185301 (2017).

[48] S. Moroz, C. Hoyos, C. Benzoni, and D. T. Son, Effective field theory of a vortex lattice in a bosonic superfluid, SciPost Phys. 5, 039 (2018).
[49] A. Bogatskiy, Vortex flows on closed surfaces, J. Phys. A: Math Theor. 52, 475501 (2019).

[50] P. Wiegmann, Quantum hydrodynamics, rotating superfluid and gravitational anomaly, J. Exp. Theor. Phys. 129, 642 (2019).

[51] D. Doshi and A. Gromov, Vortices as fractons, Commun. Phys. 4, 44 (2021).

[52] L. Landau, Theory of the superfluidity of helium ii, Phys. Rev. 60, 356 (1941).

[53] I. Dzyaloshinskii and G. Volovick, Poisson brackets in condensed matter physics, Ann. Phys. 125, 67 (1980).

[54] P. J. Morrison and J. M. Greene, Noncanonical Hamiltonian Density Formulation of Hydrodynamics and Ideal Magnetohydrodynamics, Phys. Rev. Lett. 45, 790 (1980).

[55] V. E. Zakharov and E. A. Kuznetsov, Hamiltonian formalism for nonlinear waves, Phys. Usp. 40, 1087 (1997).

[56] D. T. Son, Chiral metric hydrodynamics, kelvin circulation theorem, and the fractional quantum Hall effect, (2019), arXiv:1907.07187 [cond-mat.str-el].

[57] A. Gromov, Towards classification of fracton phases: The multipole algebra, Phys. Rev. X 9, 031035 (2019).

[58] N. Seiberg and S.-H. Shao, Exotic symmetries, duality, and fractons in 2+1-dimensional quantum field theory, SciPost Phys. 10, 027 (2021).

[59] M. Pretko, The fracton gauge principle, Phys. Rev. B 98 , 115134 (2018).

[60] N. Seiberg, Field theories with a vector global symmetry, SciPost Phys. 8, 050 (2020).

[61] H. Stark and T. C. Lubensky, Poisson bracket approach to the dynamics of nematic liquid crystals: The role of spin angular momentum, Phys. Rev. E 72, 051714 (2005).

[62] A. Nicolis, R. Rattazzi, and E. Trincherini, The Galileon as a local modification of gravity, Phys. Rev. D 79, 064036 (2009).

[63] T. Griffin, K. T. Grosvenor, P. Hořava, and Z. Yan, Scalar field theories with polynomial shift symmetries, Commun. Math. Phys. 340, 985 (2015).

[64] B. I. Halperin and D. R. Nelson, Theory of Two-Dimensional Melting, Phys. Rev. Lett. 41, 121 (1978).

[65] A. Karch and A. Raz, Reduced conformal symmetry, J. High Energy Phys. 04 (2021) 182.

[66] M. Kleman and J. Friedel, Disclinations, dislocations, and continuous defects: A reappraisal, Rev. Mod. Phys. 80, 61 (2008).

[67] S. Shankar, A. Souslov, M. J. Bowick, M. C. Marchetti, and V. Vitelli, Topological active matter, arXiv:2010.00364 [condmat.soft] (2020).

[68] T. Mura, Continuous distribution of moving dislocations, Philos. Mag. 8, 843 (1963).

[69] A. M. Kosevich, Dynamical theory of dislocations, Sov. Phys. Usp. 7, 837 (1965).

[70] B. Julia and G. Toulouse, The many-defect problem: Gauge-like variables for ordered media containing defects, J. Phys. Lett. 40, 395 (1979).

[71] A. Acharya, A model of crystal plasticity based on the theory of continuously distributed dislocations, J. Mech. Phys. Solids 49, 761 (2001).

[72] C. Fressengeas, V. Taupin, and L. Capolungo, An elasto-plastic theory of dislocation and disclination fields, Int. J. Solids Struct. 48, 3499 (2011).

[73] G. Tóth, C. Denniston, and J. M. Yeomans, Hydrodynamics of Topological Defects in Nematic Liquid Crystals, Phys. Rev. Lett. 88, 105504 (2002). 
[74] X. Tang and J. V. Selinger, Theory of defect motion in 2d passive and active nematic liquid crystals, Soft Matter 15, 587 (2019).

[75] A. Acharya, An action for nonlinear dislocation dynamics, arXiv:2104.12568 [cond-mat.mtrl-sci] (2021).

[76] W. F. Harris and L. E. Scriven, Intrinsic disclinations as dislocation sources and sinks in surface crystals, J. Appl. Phys. 42, 3309 (1971).
[77] R. deWit, Relation between dislocations and disclinations, J. Appl. Phys. 42, 3304 (1971).

[78] M. Gutkin, I. Ovid'ko, and N. Skiba, Transformations of grain boundaries due to disclination motion and emission of dislocation pairs, Mat. Sci. Eng. A 339, 73 (2003).

[79] R. C. Desai and R. Kapral, Dynamics of Self-Organized and SelfAssembled Structures (Cambridge University Press, Cambridge, 2009). 